\title{
El joven Lukács y las ciencias del espíritu
}

Diego Fernando Correa Castañeda

Universidad Nacional de Educación a Distancia,

España 


\title{
El joven Lukács y las ciencias del espíritu*
}

\begin{abstract}
Resumen: la relación que existe entre Lukács y Vattimo está determinada por la manera diferente en que se enfrentan a los postulados del materialismo, tanto histórico como dialéctico. La radicalidad del filósofo húngaro es suavizada por el filósofo italiano. Los presupuestos de absolutismo que tienen los juicios estéticos y éticos lukácsianos son tomados de una manera menos radical por Gianni Vattimo. En este artículo haremos un recorrido a través de este tipo de interacciones. Veremos que los momentos históricos a los que se enfrentaron son totalmente distintos, por lo que los resultados y las posteriores maneras de aplicación son también diferentes.
\end{abstract}

Palabras clave: Lukács, Vattimo, materialismo dialéctico e histórico, absolutismo.

\section{The young Lukács and the sciences of the spirit}

\begin{abstract}
Lukács and Vattimo is determined by the different way in which they confront the postulatesof materialism, both historical and dialectical. The radicality of the Hungarian philosopher is softened by the Italian philosopher. The presuppositions of absolutism that Lukács's aesthetic and ethical judgments have are taken in a far less radical way by Gianni Vattimo. In this article we will guide you through the type of interactions encountered. We will see that the historical moments with which they faced were totally different, so that the results and subsequent ways of application were also different.
\end{abstract}

Keywords: Lukács, Vattimo, Historical and dialectical materialism, absolutism.

Fecha de recepción: 17 de octubre de 2018

Fecha de aceptación: 12 de diciembre de 2018

Forma de citar (APA): Correa-Castañeda, D. F. (2019). El joven Lukács y las ciencias del espíritu. Revista Filosofía UIS, 18(1), doi: http://dx.doi.org/10.18273/revfil.v18n1-2019011

Forma de citar (Harvard): Correa-Castañeda, D. F. (2019). El joven Lukács y las ciencias del espíritu. Revista Filosofía UIS, 18(1), 223-233.

Diego Fernando Correa Castañeda: colombiano. Doctor en Filosofía, Universidad Nacional de Educación a Distancia.

Correo electrónico: liferco@hotmail.es

ORCID: https://orcid.org/0000-0002-9004-4300

*Artículo de reflexión derivado de investigación. 


\section{El joven Lukács y las ciencias del espíritu}

\section{Introducción}

Entre Vattimo y Lukács no es que haya existido una estrecha relación. Si en un principio Vattimo afirmó que "pienso en la filosofía en términos [...] como edificación ensayística” (Vattimo, 2012, p. 70), con ello no dejó entrever que esa era la forma artística por excelencia asumida por Lukács. Quizás, la relación entre ambos venga derivada de la oposición que establece Vattimo a los planteamientos lukácsianos, y si no hablamos de oposición, si pudiéramos hablar, al menos, de replanteamiento o debilitamiento: "Gianni Vattimo es el filósofo que ha luchado durante toda su vida contra la rigidez de la objetividad y de los absolutos que nos aprisionan" (Paterlini, 2008, p. 13).

Una de las muestras que hay en este distanciamiento la deja esclarecida el propio Vattimo en el momento en el que habla de una visita que realizó a la casa de Lukács en Budapest en el año 1969-1970, él no lo recuerda bien. La única alusión que hace sobre Lukács es que este entró en el salón de la casa llevando entre las manos unos zapatos. "Recuerdo que estábamos esperándolo en el salón y llegó con un par de zapatillas en la mano" (Vattimo, 2008, p. 109). Luego hicieron algunas alusiones referentes a Ernst Bloch. De ahí pasa Vattimo a exponer que uno de sus amigos se había decantado por la lengua húngara debido a esta visita "Gianpiero comenzó a apasionarse de este modo por el húngaro [es una pena que no haya sido por el filósofo húngaro]. No había hecho aún la tesis y, de vuelta en Italia, comenzó a escribirla en húngaro" (109-112); para continuar hablando de unos gatos que se vieron obligados a abandonar cuando se mudaron a Turín. Desde luego que la alusión a Turín está conectada con que Lukács había visitado anteriormente dicha ciudad. Este pasaje escrito en el libro de Vattimo No ser Dios, deja un poco de sinsabor; ya que uno espera que la visita 
de unos jóvenes filósofos en la propia casa de Lukács hubiera dado más frutos, y más, cuando el propio filósofo húngaro les comentó que estaba escribiendo en esos mismos momentos la obra fundamental de toda su vida como filósofo: "Se refirió [Lukács] al libro que estaba escribiendo, La ontología del ser social" (Vattimo, 2008, p. 110).

Una vez hecha esta corta y extraña introducción podremos dar paso a los diferentes escenarios en los que se desenvolvió la obra de nuestros dos autores. La crisis que se estaba desarrollando en Europa en el momento en el que Lukács simpatizaba con las ciencias del espíritu era muy distinta de la crisis de valores políticos y democráticos en la que se desarrolló la izquierda hermenéutica, la cual, además, estaba altamente impregnado de la noción de secularidad, como lo deja claramente expuesto Vattimo en su obra Creer que se cree (1996, pp. 49ss.). Es más, podría decirse que el comunismo hermenéutico está precisamente en contra del absolutismo filosófico que profesaba Lukács. De ahí que, ante la fuerte imposición lukácsiana de una visión radical de la realidad, Vattimo hable más bien de un "debilitamiento de la realidad" (Vattimo, 2013, p. 26) o de "[...] asumir una perspectiva 'no absolutista'" (Vattimo, 2012, p. 67). Por su parte, Lukács, en muchas partes de su ingente obra, lo deja claro a cada paso:

La relación real entre la misión social y la obra consiste más bien en que cuanto más orgánica es la consumación estética inmanente de una obra de arte, tanto más capaz es ésta de cumplir la misión social que le ha dado vida. Esta concepción -la única correcta- de las interacciones entre la individualidad de la obra y la misión social (Lukács, 1967, p. 368).

En el primer tomo de la misma Estética, en la Introducción, vuelve otra vez el mismo pensamiento:

En los clásicos mismos no se encuentra rastro alguno de esa metafísica contraposición entre lo viejo y lo nuevo. La relación entre ambos se presenta más bien en las proporciones producidas por el desarrollo histórico-social mismo al hacer manifestarse la verdad. El aferrarse a este método, único correcto, es acaso para la estética aún más importante que en otros terrenos (Lukács, 1967, p. 18).

Antes de ver la incursión del Lukács joven en el campo de las ciencias del espíritu, sería importante hacer alguna aclaración sobre los primeros pasos seguidos por este y, también, alrededor de las posibles similitudes entre las dos crisis. Quizás el rasgo más importante en la vida de Lukács es que se estaba desarrollando en un período de grandes cambios:

La vida intelectual alemana de esos años anteriores a la primera guerra mundial se centraba en varios procesos importantes: la disolución de la escuela neokantiana [de la que él iba a formar parte], la aparición de la 
fenomenología y el fortalecimiento de las corrientes irracionalistas e intuicionistas [como] últimas prolongaciones de movimiento romántico (Lichtheim, 1973, p. 34).

Lo que ocurre es que, a diferencia de Vattimo, para quien la izquierda debe ser reelaborada desde el pensamiento débil, o sea, plantear ya desde la distancia un nuevo principio, reinterpretando lo anterior, cuando no abandonar directamente la línea absolutista dejada por Hegel, continuada por Marx y desarrollada por Lukács: "creo que el pensamiento débil es la única alternativa al hegelianismo" (Vattimo, 2012, p. 65); por el contrario, para Lukács, las cosas eran muy distintas. A él le tocaba adoptar la posición del que está ante un auténtico cambio histórico, como, por ejemplo, la noción de que ya ha llegado el momento para que una nueva clase emergente sea la que tome el poder de su propio destino. Estos acontecimientos fueron los causantes de que Lukács se viera en la obligación de tomar partido y de radicalizar su postura:

Sólo ahora ha llegado el momento de desarrollar el materialismo histórico como un método científico precisamente porque el proletariado se ha apoderado del poder. Con el poder, el proletariado dispone de las fuerzas físicas e intelectuales sin las cuales no podría alcanzar su fin, fuerzas que la vieja sociedad nunca habría puesto a su servicio (Lukács, 1978, p. 88).

\section{La búsqueda de Lukács}

El camino que emprende Lukács, en la búsqueda de las respuestas que necesita la nueva situación social, lo lleva a explorar los momentos históricos más destacados para poder esclarecer el conflicto ético y estético con el que se estaba enfrentando. En este sentido, la fecha que marca el cambio más radical es la Revolución Democrática de 1848, ya Lukács pone esta fecha como el punto de inflexión en el que se presenta la gran ruptura en la forma con la que el hombre se enfrenta al mundo; quizás uno de los hechos más preocupantes sea que: "la filosofía alemana no ha podido ya reponerse a partir de 1848" (Lukács, 1970, p. 9). Esta amalgama de hechos condujo al advenimiento de la barbarie ante la que inevitablemente había que tomar partido.

Las implicaciones éticas y estéticas que traían los cambios de los nuevos tiempos impelían a la creación de diferentes presupuestos para el nacimiento de un nuevo mundo. La nueva clase social floreciente tenía que ser dotada de unos nuevos estatutos, tanto de conducta como de creación artística. Es en este momento en el que Lukács, en contraposición con Vattimo, toma el camino radical de demoler las viejas estructuras y crear unas nuevas. No se trata de maquillar o ablandar lo que ya está establecido, por miedo quizás a absolutizar ideológicamente lo dado, sino de destruir lo anterior y extraer lo mejor de la nueva producción, tanto ética 
como estética. Solo así podemos entender esta sentencia lukácsiana: "La Teoría de la novela no es conservadora, sino destructora" (Lukács, 1999, p. 42).

El ambiente en el que se estaban desarrollando los acontecimientos ya tenía varias vías de penetración: Era la época en la que se estaba dando la disolución del romanticismo. Hegel ya había dicho que lo cotidiano entraba a formar parte del arte (2006, p. 361). Lukács descubre, en el campo de la moral, que la disolución del neokantismo se debe a que el Sollen kantiano estaba llegando a su fin, es decir, la moral kantiana se corrompe en la forma de una burguesía que se niega a desaparecer, lo que desemboca a larga en la Primera Guerra Mundial; este conflicto demostró que el deber-ser no implicaba la unión de los pueblos sino la lucha entre ellos. La Revolución Rusa se erige en el acontecimiento, visto desde el distante misticismo oriental, de donde brotarán los nuevos parámetros éticos con los que se tiene que dirigir la conducta humana. Será de ahora en adelante la bondad dostoievskiana, oculta detrás de los personajes de sus obras, la que guiará a la humanidad. Valga recordar en este sentido que esto influyó de manera notable en el propio desarrollo de la vida de Lukács, referente a su relación con Irma Seidler y Sorel Kierkegaard. El fracaso de la revolución proletaria en Alemania será el otro acontecimiento fundamental para entender esta interacción de adopción y rechazo.

\section{Acercamiento a las ciencias del espíritu a través del neokantismo}

Llevado por lo profundo de las crisis, Lukács emprendió durante su juventud gran número de viajes por diversas ciudades europeas —el ser hijo de un banquero rico y próspero se lo permitió-, llegando a vivir luego en algunas de ellas: Florencia, Berlín y desde luego Heidelberg. Fue en esta última en donde tuvo contacto con gran parte de la intelectualidad alemana, más que todo con personalidades de la talla de Max Weber, Emil Lask y Georg Simmel. Por aquel entonces:

Había dos escuelas principales de neokantismo: la escuela de Marburgo, cuyos representantes punteros eran Cohen y Natorp [interesados por la filosofía, el pensamiento no debe ser responsable solo de la forma sino también del contenido. Lukács rechaza esto. No ve cómo puede ser posible que "el problema de la realidad podría ser derivado a una categoría de la consciencia], y la escuela de Heidelberg... cuyos principales representantes eran Windelband y Rickert [interés por problemas de historia y cultura] (Parkinson, 1973, p. 11).

Pero fueron más que todo Simmel, Lask, Weber y Dilthey "conviene no perder de vista que quien primero abrió los ojos a Lukács sobre la radical diferencia existente entre la ciencia natural y la historia fue Dilthey" (Lichtheim, 1973, p. 43) los que mayor influencia ejercieron sobre él. Lukács asistió a las clases de Simmel 
y de Weber cuando era estudiante tanto en Berlín como en Heidelberg. Estamos hablando de los años 1910-1911. No creo que se le deba reprochar a Lukács que haya estado transitando por la filosofía kantiana cuando era joven, dado que, al fin y al cabo.

En aquel período el neokantismo era la tendencia dominante en la filosofía del mundo de habla alemana, en el que estaban incluidas las clases cultas del Imperio Austro-Húngaro, y, en consecuencia, nada tiene de sorprendente el que Lukács cayese bajo la influencia de un tipo de filosofía neokantiana (Parkinson, 1973, p. 11).

También conviene decir que recibió de ella una formación rigurosa y disciplinada, lo que le permitió a la larga obtener un mayor conocimiento de los sistemas filosóficos imperantes en aquel momento. Fruto de esta etapa nació su polémica obra Historia y conciencia de clase (1923). En este sentido sería también sano recordar que este paso por la filosofía neokantiana le ayudó a penetrar de una forma más segura en los sistemas kantiano y hegeliano, quienes le habían causado problemas de comprensión tanto a Engels como a Lenin, lo que más tarde le acarreó muchos inconvenientes a Lukács. Aunque él ya tenía algunas nociones de marxismo, seguía prefiriendo la filosofía neokantiana:

[Lukács] leyó el Manifiesto Comunista cuando era todavía estudiante de bachillerato, y durante su licenciatura estudió varias obras de Marx y Engels: él menciona en particular el primer volumen de El Capital de Marx, y añade que la influencia de Marx sobre él estuvo al principio limitada a la economía y la sociología; no aceptaba el materialismo marxista, que le parecía superado por la teoría neokantiana del conocimiento (Parkinson, 1973, p. 11).

El período es muy rico y variado, proclive para la proliferación de todo tipo de hipótesis y teorías, tanto científicas como filosóficas. Dentro de este ambiente ambivalente y cambiante surge la necesidad de volver a determinar cuáles son verdaderamente las reales condiciones del conocimiento. Inmerso en este debate se encuentra la disputa entre las ciencias naturales y las llamadas ciencias del espíritu, emanadas precisamente de esta candente confrontación, surge la necesidad de determinar cuál es el objeto de investigación de cada una de ellas; las ciencias naturales evidentemente tienen su objeto de estudio bien delimitado, ¿pero las del espíritu? "La posibilidad del experimento en las ciencias positivas facilita hablar de objetos, mientras que en el caso de la filosofía es más difícil decir si efectivamente hay un objeto o no" (Vattimo, 2012, p. 72). Las ciencias naturales se han apoderado del conocimiento objetivo de la realidad:

Si ellas tienen razón, la filosofía es una superviviente ideológica que ya no tiene razón de ser, y de la cual hay que liberarse. Pero si, por el contrario, la filosofía aporta realmente verdades acerca de la naturaleza del hombre, 
entonces toda tentativa de eliminarla altera necesariamente la comprensión de los hechos humanos. En este caso, las ciencias humanas debes ser filosóficas para ser científicas (Goldmann, 1975, pp. 7-8).

En toda esta amalgama se: «Abre un período que se distingue por la creciente intensidad de la lucha de clases y su reflejo casa vez mayor en el mundo de la cultura, un creciente desesperar de la razón y un creciente sentimiento de la necesidad de una Weltanschauung o mito, para hacer soportable la vida» (Hodges citado en Parkinson, 1973, p. 113). Pero, de todas maneras, lo que más importaba para el mundo filosófico del momento era que: «Cuando el renacimiento neokantiano de la década de 1870 dio nueva vida a la filosofía, lo hizo dando por supuesto que en lo sucesivo el filósofo iba a renunciar a la búsqueda de conocimientos susceptibles de quedar ocultos para el científico» (Lichtheim, 1973, p. 52).

\section{Distanciamiento de las ciencias del espíritu}

Lukács era totalmente consciente de la situación, por este motivo abandona la filosofía neokantiana y podría decirse que con ella toda la corriente de la Geisteswissenschaften. Son varias las causas de dicho distanciamiento, pero básicamente se centran en tres grandes temas: el primero, y más importante, es que la filosofía neokantiana tiene un carácter a-histórico, además de que deja abierta una brecha entre los valores intemporales y la realización histórica de dichos valores.

Lukács abandonó pronto el neokantismo, por lo que él consideraba como carácter a-histórico de aquella forma de pensamiento. Rickert y su escuela habían abierto una brecha entre los valores intemporales, por una parte, y, por la otra, la realización histórica de los valores. En La Teoría de la Novela [...] Lukács rechazó ese modo de ver, a favor de una historización de las categorías estéticas. Esa idea derivaba de Hegel, y, en efecto, Lukács considera La Teoría de la Novela como marcando su transición de Kant a Hegel (Parkinson, 1973, p. 13).

Si el primer motivo del distanciamiento estaba ligado a presupuestos históricos, toda la posición adoptada ante el devenir histórico de la humanidad; el segundo motivo tiene, a su vez, otras variantes. Esta vez se trata de la disyuntiva ante la que se tenía que enfrentar el sujeto.

Lukács entraba de forma original en escena en un momento en el que se creía muy extendidamente que la única elección que se ofrecía a quien no aceptaba la metafísica tradicional ni la fe religiosa era la planteada entre el positivismo de las ciencias empíricas y el vitalismo [...] la insatisfacción ante esta alternativa era muy frecuente en los medios académicos [...] de ahí la fascinación por Dilthey [...] lo que la Geisteswissenschaft venía a explicar en última instancia era la identidad del espíritu del propio pensador con el 
Espíritu cuyas manifestaciones se exponen ante nuestros ojos en la historia (Lichtheim, 1973, p. 51).

Esto es, o bien te plegabas al positivismo o bien a las ciencias del espíritu, y no nos olvidemos del vitalismo. Ante esta disyuntiva, Lukács, antes de conocer el marxismo, siente la atracción del llamativo sistema diltheyano.

Lo que la Geisteswissenschaft venía a explicar en última instancia era la identidad del espíritu del propio pensador con el Espíritu cuyas manifestaciones se exponen ante nuestros ojos en la historia" dice Lichtheim, o lo que es lo mismo "[es] en el acto de Verstehen (comprensión) de la Erlebnis (de la vivencia) en virtud del cual el pensador individual venía a trascender el nivel psicológico, reconstruyendo el significado del mundo objetivo" (Lichtheim, 1973, p. 56).

Piénsese solo en la diferencia que marca Dilthey entre la psicología explicativa —Lukács la ve insuficiente, ya que no explica la conexión entre el mundo físico y psíquico- - y la psicología interpretativa, con esta última Dilthey tratará de llegar hasta el final. Hablando de la hermenéutica, de la aplicación sistemática del "comprender", que tiene "un carácter adivinatorio, sin llegar nunca a una certeza demostrativa". Lukács habla de teoría aristocrática del conocimiento. Pero, claro, este no podía estar de acuerdo con esto, aquí estamos ante el tercer motivo del distanciamiento lukácsiano de las ciencias del espíritu. Para Lukács este enredo pseudo-especulativo lo resolvía de manera clara la dialéctica, tanto materialista como histórica.

En este sentido, hay que recordar además que Vattimo estaba en contra de postular a la filosofía como una herramienta del conocimiento absoluto, esto es, con sus pretensiones de método universal de validez. Vattimo quizás comparta con Lukács, y con las escuelas del materialismo, los presupuestos históricos, pero no los dialecticos. Podemos ver aquí también un motivo más de la distancia que separa a Vattimo de Lukács. "Sobre todo no creo que la filosofía sea una ciencia en el sentido estricto de la palabra" (Vattimo, 2012, p. 55), pero si ve por el contrario su aplicación en el terreno de la historia. "Se puede quizás hablar de cientificidad en la historia de la filosofía, porque en cuanto historia de algo mantiene puntos de referencia más o menos objetivos" (55). Si antes había marcado distancias con la escuela neokantiana, ahora le había llegado el turno, al —entre tanto reproche - admirado Dilthey. Pero la separación es más dolorosa, no solo por presupuestos netamente teóricos, sino porque Lukács cree ver también en Dilthey a un precursor del nazismo:

En Wilhelm Dilthey ve Lukács la fuente indiscutible de aquella Lebensphilosophie que es la forma típica del irracionalismo en el período imperialista. No obstante, del propio Dilthey habla siempre Lukács con un notable respeto. Reconoce la profundidad de su erudición, aun cuando 
estuviese falto de la luz de la teoría marxista para obtener la verdadera interpretación de los hechos. Reconoce su honrado deseo de escapar del subjetivismo y del irracionalismo, aunque de hecho no consigue escapar de uno ni de otro. Reconoce que Dilthey se habría horrorizado si hubiera podido prever el nazismo, aunque de hecho sus escritos cuenten entre las causas que hicieron posibles las doctrinas nazis (Dilthey citado en 1973, p. 115).

Aparte de esta supuesta directriz fascista oculta detrás de la obra diltheyana, Lukács lleva también a cabo contra Dilthey las mismas críticas que dirigió contra Nietzsche, al considerar que, su ignorancia de los fenómenos económicos, en los que se estaba desarrollando su filosofía, era la culpable en última instancia del desvío de su sistema.

Como Dilthey no sospecha la naturaleza de la base económica de la sociedad, sino que solamente observa las sobreestructuras culturales que encuentra reflejadas en sus fuentes escritas [aquí está una de las fuentes y de las conexiones con la hermenéutica], sin sospechar nunca cómo la consciencia superficial oculta y distorsiona la realidad subyacente, su descripción, aunque vívida, y perfectamente honrada dadas las fuentes en que se basa, es subjetiva en lo que selecciona, en lo que subraya y en lo que interpreta (Dilthey citado en 1973, p. 118).

Recordar que, en este sentido, fue este desconocimiento de la base, o la interacción de la producción social, lo que obligó a Nietzsche a adoptar las mismas críticas que lanzaban los románticos contra el capitalismo, —aparte de su vertiente inclinada hacia la naturaleza—, esto es, nada de auténtico conocimiento de la realidad material que se desarrolla delante de sus ojos, sino simples críticas superficiales de un mal mucho más grande, pero que desafortunadamente para Nietzsche era invisible.

\section{Conclusión}

Las posiciones adoptadas por los diferentes sistemas filosóficos ante la realidad histórica han tomado todo tipo de variantes. En primer lugar Schelling había preferido volver hacia atrás, antes de que se hubieran presentado las mismas contradicciones del sistema político y económico destructor de todo lo humano, y con ello impregnar toda la escuela de los románticos; por su parte Hegel había optado por hacer un reconocimiento de los hechos mismos y emprender un análisis lógico sobre los motivos de la problemática; por otra parte otros prefirieron tomar posiciones utópicas y buscar otras vías de salvación; por último el marxismo se planteó la cuestión de la eterna interpretación que siempre se habían hecho sobre los acontecimientos y emprendió la tarea de su transformación. En esta contradictoriedad se inscribe el debate en torno a nuestros dos autores: Lukács y Vattimo. Lukács toma el camino del marxismo y opta por ahondar en la 
contradictoriedad haciendo para ello un recorrido histórico que lo llevó desde el mundo clásico griego hasta el nuevo resurgir de lo heleno en suelo ruso. Por su parte, Vattimo parece querer dejar aparcado el debate: ni regresar antes de que se hubieran presentado las contradicciones, ni lograr un reconocimiento de las mismas, ni declinarse por visiones utópicas, ni plegarse al marxismo y tratar de avanzar en la misma dirección marcada por aquel, ni seguir a Lukács destruyendo los primados éticos y estéticos para poder dejar espacio al nacimiento de nuevos mundos. No, Vattimo opta por aplicar la medicina a cuenta gotas, suministrar el antídoto, pero pausadamente, suavemente. Podríamos decir que aplicando una "cura de adelgazamiento del sujeto" (Vattimo, 1987, p. 46).

\section{Referencias}

Goldmann, L. (1972). Para una sociología de la novela. Madrid: Editorial Ayuso.

Hegel, G, W, F. (2006). Estética. Madrid: Abada.

Lichtheim, G. (1973). Lukács. Barcelona: Grijalbo.

Lukács, G. (1970). Realistas alemanes del siglo XIX. Barcelona: Grijalbo.

Parkinson, G, H, R, ed. (1973). Georg Lukács. Barcelona: Grijalbo.

Vattimo, G. (1987). El fin de la modernidad. Nihilismo y hermenéutica en la cultura posmoderna. Barcelona: Gedisa.

Vattimo, G. (2008). No ser Dios. Barcelona: Paidós.

Vattimo, G. (2012). Vocación y responsabilidad del filósofo. Barcelona: Herder.

Vattimo, G. (2013). De la realidad. Fines de la filosofía. Barcelona: Herder. 